\title{
A brittle fracture criterion for PMMA V-notches tensile specimens based on a length-enriched eXtended Finite Element approach
}

\author{
E. Benvenuti, R.Tovo, P. Livieri \\ Università di Ferrara
}

\begin{abstract}
A criterion for the prediction of the static failure loads in tensile PMMA specimens with sharp notches is presented. The proposed criterion is based on a regularized version of the eXtended Finite Element Method (XFEM), which has been previously applied to concrete-like materials. The main feature of the proposed approach is that the cracking process is not treated as a local process, but it is modeled by assuming that macro-cracks stem from the interaction of micro-cracks within a finite width process zone. The case of a brittle materials with thin process zone is tackled by assuming one layer of enriched finite elements. Preliminary results concerning PMMA specimens subjected to mode-one loading are presented.
\end{abstract}

KEYWORDS. Static failure criteria; PMMA; XFEM.

\section{INTRODUCTION}

$\mathrm{S}$ harp notches in tensile structural elements trigger crack initiation and influence the global load bearing capacity. Hence the necessity of formulating reliable and effective static failure criteria for structures with sharp notches emerges. In particular, if the case of notched components made of brittle materials is considered, criteria based on the evaluation of the local maximum value of a stress scalar fail, owing to the presence of infinite values of the stress field components.

As argued by Neuber [1], effective static fracture criteria can be introduced by assuming that crack initiation is governed by volume, or surface, averages of the local stress scalar field, which is most representative of the expected failure mode. For instance, Seweryn [2] proposed a criterion based on the evaluation on the maximum value of the stress scalar, which is obtained by averaging the stress component normal to the crack direction along the crack path. A further extension of this approach can be envisaged in the critical distance criteria proposed by Taylor et al. [3].

The introduction of volume averages of stress scalars can be alternatively interpreted as the adoption of a non-local fracture criterion, where the critical value of a certain scalar field at a point depends not only on the local value of that field at the current point, but also on the values that the scalar field reaches at the surrounding points [4, 5]. The non-local continuum concept has been especially exploited in the numerical analysis of components made of elasto-damaging materials. Specifically, two variants of non-local models have been intensively used: integral models and gradient models. Inspired by the approach proposed by Peerlings et al. [6], Tovo et al. [7, 8] proposed an implicit gradient static failure criterion which is very reliable, as it can be easily implemented by using standard tools for solving partial differential equations within usual finite element codes. An advantage of the implicit gradient static failure criterion is that the nonlocal equivalent stress can be obtained over the entire spatial domain without a priori assumption of the position of the critical points [7].

An alternative approach to overcome the stress singularity at the crack tip is represented by the adoption of a cohesive crack model [9-11]. In the cohesive crack model, a traction-separation law is introduced along the crack path. The 
cohesive crack approach can be extended to rounded notches and makes it possible to deal with non-linear materials. A limitation of the cohesive-crack models is that the crack path has to be placed along the element boundaries. Therefore, the crack geometry depends on the mesh topology. This drawback can be overcome by adopting the eXtended Finite Element method (XFEM) method which has been developed by Belytschko and coworkers [12]. XFEM makes it possible to consider crack paths, which are embedded within the finite elements. XFEM is based on the partition of unity property of the polynomial shape functions, which are used in standard finite element analyses. For instance, in the case of crack problems, where a displacement discontinuity has to be simulated, the standard piecewise continuous approximation of the displacement field is enriched by additional terms, which contain the Heaviside function. More recently, enhanced XFEM approaches have been proposed were the truncated series of William's expansion of the displacement field are considered as enrichment functions [13-15].

In quasi-brittle materials, experimental evidence shows that macro-cracks stem from the interaction if micro-cracks distributed within a zone of finite width, called fracture process zone (e.g. [16]). Benvenuti et al. [17-19] have proposed a modified XFEM approach which is based on the use of regularized enrichment functions. In particular, the discontinuous step function is replaced by a regularized Heaviside function where a regularization length is introduced. A cohesive-like stress field emerges along the crack path which is always non singular and smooth. The smoothing effect on the stress field increases for increasing values of the regularization length. In the present study, a static failure criterion based on the regularized XFEM approach is applied to the analysis of the tensile PMMA specimens previously proposed by Seweryn [2]. The pros and cons of the present approach are discussed.

\section{BASICS OF THE PROPOSED EXTENDED FINITE ELEMENT FORMULATION}

L

et us consider a tensile specimen with a sharp notch. The displacement field is supposed to exhibit a jump $\left\|\boldsymbol{u}^{+}-\boldsymbol{u}^{-}\right\|$across a line $S_{d}$ starting from the notch tip. The vector field $\mathbf{n}$ normal to $S_{d}$ is introduced.

Let us discretize the geometry through $N_{e}$ finite elements with $N_{n}$ nodes, and introduce the regularized Heaviside function

$$
H(x)=\operatorname{sign}(s(x))\left(1-\exp ^{-|s(x)| / \rho}\right)
$$

where $s(\boldsymbol{x})$ denotes the signed distance function from the displacement discontinuity defined as $s(\boldsymbol{x}):=|\overline{\boldsymbol{x}}-\boldsymbol{x}|$ if $|\overline{\boldsymbol{x}}-\boldsymbol{x}| \cdot \boldsymbol{n}(\boldsymbol{x}) \geq 0$ and $s(\boldsymbol{x})=-|\overline{\boldsymbol{x}}-\boldsymbol{x}|$ if $|\overline{\boldsymbol{x}}-\boldsymbol{x}| \cdot \boldsymbol{n}(\boldsymbol{x})<0$. A picture of the regularized Heaviside function is given in Fig. 2 for various $\rho$.

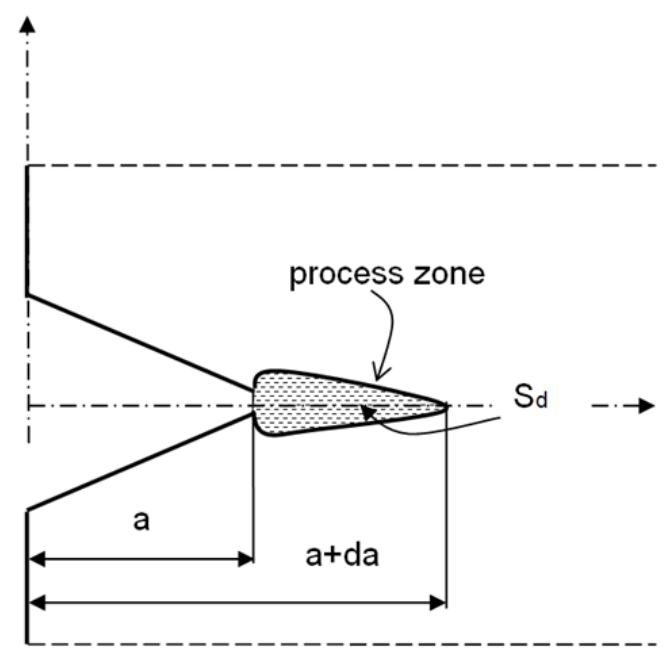

Figure 1: Tensile element with a notch.

According to the regularized XFEM formulation recently proposed by Benvenuti et al. [17-19], the displacement field can be expressed as 


$$
u_{\rho}(x)=\sum_{i=1}^{n} N_{i}(x) v_{i}(x)+\sum_{i=1}^{n} N_{i}(x) H_{\rho}(x) a_{i}(x)
$$

where: $N_{i}$ represents the $\mathrm{i}$-th standard polynomial shape function of the usual finite element approximation, $\mathbf{v}_{\mathrm{i}}$ is the $\mathrm{i}$-th standard degree of freedom representative of the continuous part of the displacement field. Finally, $\boldsymbol{a}_{i}$ denotes the i-th enriched degree of freedom.

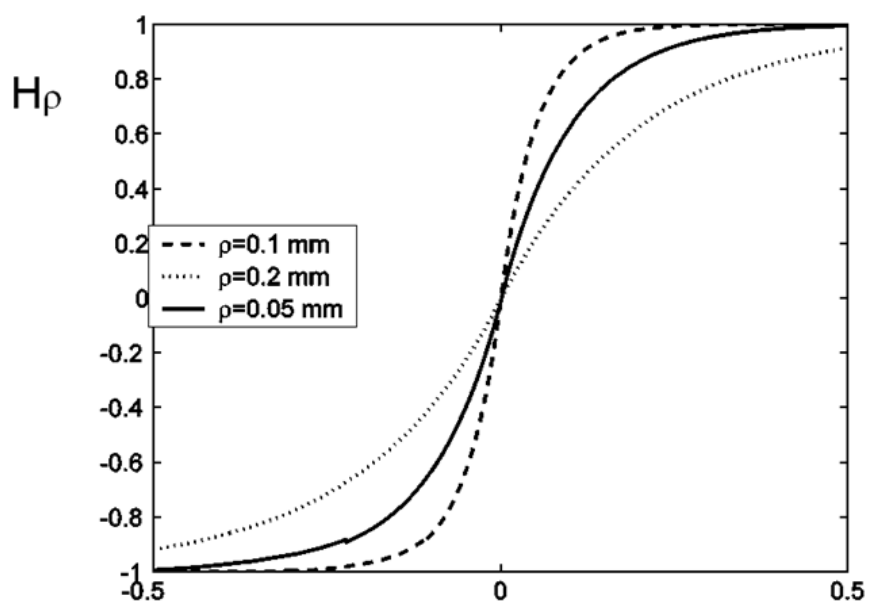

Figure 2: Regularized Heaviside function and corresponding gradient for various $\rho$.

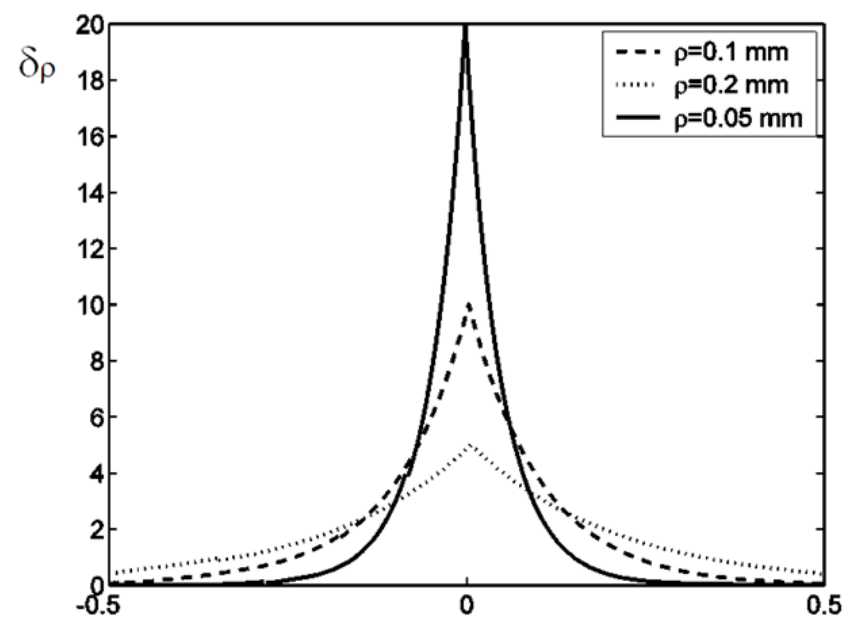

Figure 3: Micro-cracks density function $\delta_{\rho}$ obtained by differentiation of $H_{\rho}$.

After collecting the shape functions into the matrix $\boldsymbol{N}$ and the degrees of freedom $\mathbf{v}_{\mathbf{i}}$ and $\boldsymbol{a}_{\boldsymbol{i}}$ into vectors $\boldsymbol{V}$ and $\boldsymbol{A}$, the displacement field writes

$$
u_{\rho}(x)=N(x) V+N(x) H_{\rho}(x) A
$$

The compatible strain is calculated by differentiation of displacement $\boldsymbol{u}_{\boldsymbol{\rho}}$ in the following way

$$
\nabla u_{\rho}=\nabla N(x) V+\nabla N(x) H_{\rho}(x) A+\delta_{\rho}(x)(n \otimes N(x) A)
$$

where $\nabla H_{\rho}=\delta_{\rho} \mathbf{n}$ and

$$
\delta_{\rho}(x)=\exp (-|s(x)| / \rho)
$$


In particular, the form of the function $\delta_{\rho}$, which is displayed in Fig. 4, reproduces the distribution of micro-cracks within the process zone which is often recognized in quasi-brittle materials [16]. The presence of a regularization parameter $\rho$ introduces a characteristic length within the present XFEM formulation.

After introduction of the compatibility operator $\boldsymbol{B}=\nabla \boldsymbol{N}$ and $\overline{\boldsymbol{N}}$ such that $\overline{\boldsymbol{N}} \boldsymbol{A}=(\mathbf{n} \otimes(\mathbf{N}(\mathrm{x}) \mathbf{A}))$, three distinct strain fields

$$
\begin{aligned}
& \varepsilon(x)=B(x) V \\
& \varepsilon_{\rho}(x)=H_{\rho}(x) B(x) A \\
& \varepsilon_{\rho}^{c}(x)=\delta_{\rho}(x) \bar{N}(x) A
\end{aligned}
$$

can be introduced. They are kept distinguished because they play a different mechanical role within the model. In particular, the stress field $\boldsymbol{\varepsilon}$ is the standard strain field in the absence of the discontinuity. The strain field $\boldsymbol{\varepsilon}_{\rho}$ is related to the spatial variation of the jump intensity, while $\boldsymbol{\varepsilon}_{\boldsymbol{\rho}}^{\mathbf{c}}$ represents the localized strain field resulting from the micro-cracks distributed within the process zone.

Independent stress fields are associated with each strain field by means of the following constitutive laws

$$
\begin{aligned}
& \sigma(x)=(1-D) E B(x) V \\
& \sigma_{\rho}(x)=H_{\rho}(x)(1-D) E B(x) A \\
& \sigma_{\rho}^{c}(x)=\left(1-D_{c}\right) E_{c} \bar{N}(x) A
\end{aligned}
$$

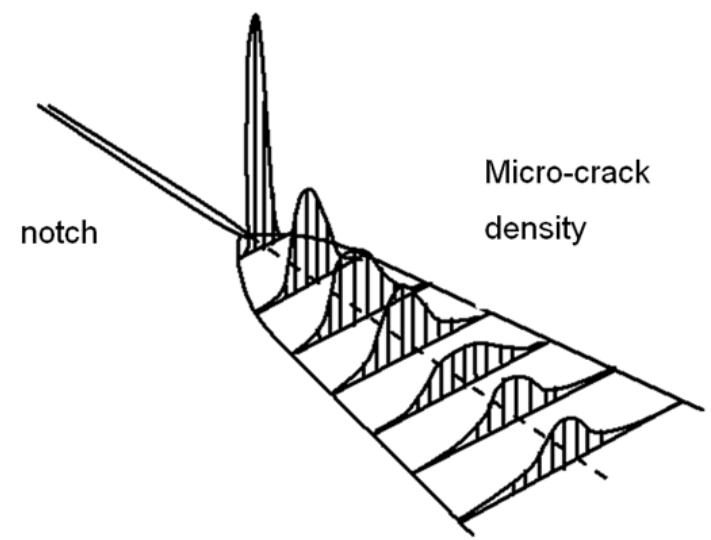

Figura 4: Microcrack density distribution according to Mihashi and Nomura [16].

where $\mathrm{D}_{\text {and }} \mathrm{D}_{\mathbf{c}}$ are isotropic damage scalar parameters which govern the degradation of the elasticity matrices $\mathbf{E}$ and $\mathbf{E}_{\mathrm{c}}$. A detailed explanation of the transition strategy from a continuum damage description to the regularized discontinuous description can be found in Benvenuti (2011). The key point of the transition procedure is that, as soon as the bulk damage parameter $D$ has reached a critical value, $D_{c r}$, the damage $D_{c}$ is activated. In particular, for a loading history described by the pseudo-time parameter $\mathrm{t}$, the evolution of the damage parameter $D_{c}$ is governed by the following loading unloading conditions

$$
\varphi=\sigma_{e f f}-r, \varphi \leq \dot{0}, \dot{D}_{c} \geq 0, \quad \dot{\varphi} \dot{D}_{c}=0
$$

The effective stress scalar $\sigma_{e f f}$ selects the positive value of the maximum principal stress $\bar{\sigma}_{\max }$ of the stress field $\boldsymbol{\sigma}_{\boldsymbol{\rho}}^{\boldsymbol{c}}$

$$
\sigma_{e f f}=\left\langle\bar{\sigma}_{\max }\right\rangle
$$


The damage is calculated as

$$
D_{c}=\max \left\{D_{c r}, 1-\frac{q(r)}{r}\right\} \quad r=\max \left(\boldsymbol{r}_{0}, \max \left\{\sigma_{e f f}(t)\right\}\right) \quad t \in[0, \infty)
$$

where $\mathrm{q}$ is an internal variable defined as

$$
\boldsymbol{q}(\boldsymbol{r})=\boldsymbol{r}_{0} \exp \left(-2 \boldsymbol{H}_{s} \frac{\boldsymbol{r}-\boldsymbol{r}_{0}}{\boldsymbol{r}_{0}}\right) \quad \boldsymbol{r} \geq \boldsymbol{r}_{0}
$$

and $r_{0}$ is an initial threshold, which is set equal to the maximum tensile stress, which can be deduced from the experiments.

The variational formulation which has been adopted makes it possible to recover standard cohesive model for vanishing regularization length. Moreover, the ductility of the computed structural paths turns out being proportional to $\rho$ itself. Details can be found in previous References [17-19].

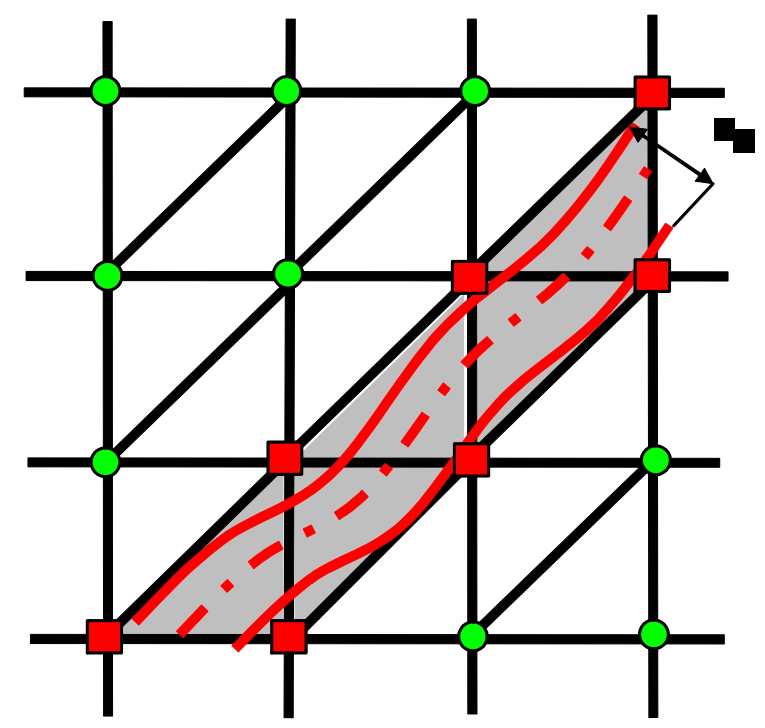

Figura 5: Thin process zone enrichment.

The enrichment strategy is general: it can be applied to both the cases of thin and thick process zone. More precisely, the width of the enrichment layer can be larger than a single layer of finite elements. In this case, the model can be seen as an XFEM with cohesive crack. Alternatively, the width of the enriched volume can be larger than a single layer of finite elements, similarly to what happens in a non-local integral model. The enrichment strategy has been thoroughly discussed in a previous Reference [17]. In the present preliminary analysis, the analysis is applied to very brittle materials such as the PMMA. To this purpose, only one layer of finite elements has been enriched, as shown in Fig. 4, where represents the width of the truncated support of function $\delta_{\rho}$. In the present analyses contributions of Gauss points placed at distances larger than $20 \rho$ were neglected. Moreover, the nodes marked with a red square indicate the enriched nodes, while the nodes which are not enriched are indicated by a green circle. The enriched elements have been indicated in light gray.

\section{NUMERICAL RESULTS}

$\mathrm{T}$ he PMMA tensile specimen experimentally tested by Seweryn [2] has been considered (Fig. 6). The width W is 109 $\mathrm{mm}$, while the thickness is equal to $4 \mathrm{~mm}$. The notch depth a $=27 \mathrm{~mm}$. In view of the symmetry of both geometry and loading, only one half of the specimen was studied. A plane stress two-dimensional analysis was performed. The constitutive law for the PMMA, which is shown in Fig. 7 for the one-dimensional case, has been adopted according to the material parameters given in Seweryn (1994). In particular, Young modulus E=3200 MPa, Poisson coefficient equal to 0.4 and a maximum effective stress $105 \mathrm{MPa}$ have been taken. 


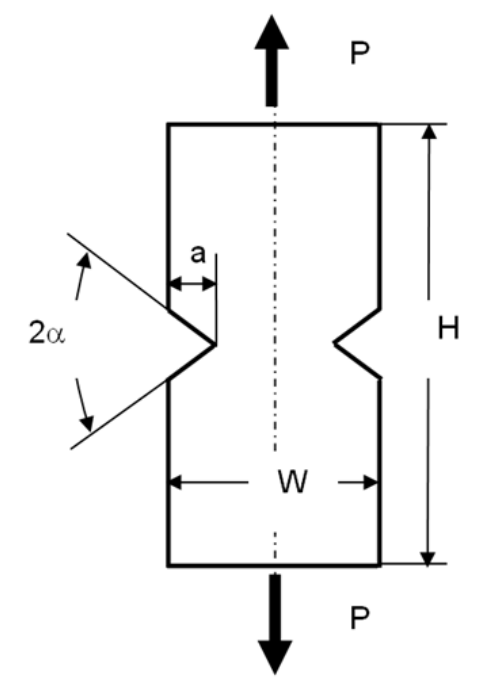

Figure 6: Tensile specimen geometry after Seweryn (1994).

The limit tensile stress which was indicated by Seweryn is $105 \mathrm{MPa}$. As soon as the bulk reaches the critical damage $D_{c r}=0.01$, the continuous-discontinuous transition is activated.

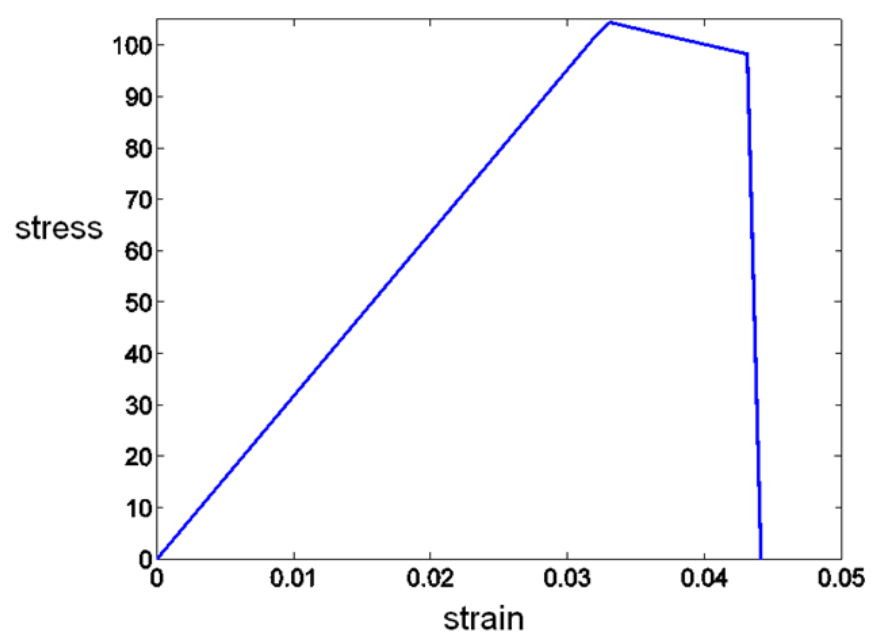

Figure 7: Constitutive law of the PPMA which has been adopted in the numerical tests.

The meshes which were adopted are refined in the central zone as shown in Fig. 8. A minimal value of the finite element size $\mathrm{h}=0.1 \mathrm{~mm}$ was used for all opening angles (semi-opening angle $\alpha=10,20,30,40,50,60,70$ degrees). In the case of a tensile plate made of a softening materials, it was shown in Benvenuti [19], that the optimal value of the parameter $\rho$ which leads to optimal convergence is $\rho=1 / 13.33 \mathrm{~h}$ which in the present case leads to $\rho=0.0075 \mathrm{~mm}$.

In order to check the ability of the method to effectively approximate the experimental failure loads, a unique value of the ratio between the minimal mesh size $\mathrm{h}$ and the parameter $\rho$ was adopted for all meshes. In order to identify the optimal value of the regularization length $\rho$, the following values have been adopted: $\rho=0.0001,0.002,0.0075,0.01 \mathrm{~mm}$. The critical failure load versus half the opening angle of the notch are displayed in Fig. 8. In particular, the value of $\rho=0.0001$ $\mathrm{mm}$ leads to divergent results, while the value $\rho=0.0075 \mathrm{~mm}$ leads to the best approximation. Fig. 10 shows the computed failure loads for various opening angles and the experimental values from Seweryn [2] by using $\rho=0.0075 \mathrm{~mm}$. 


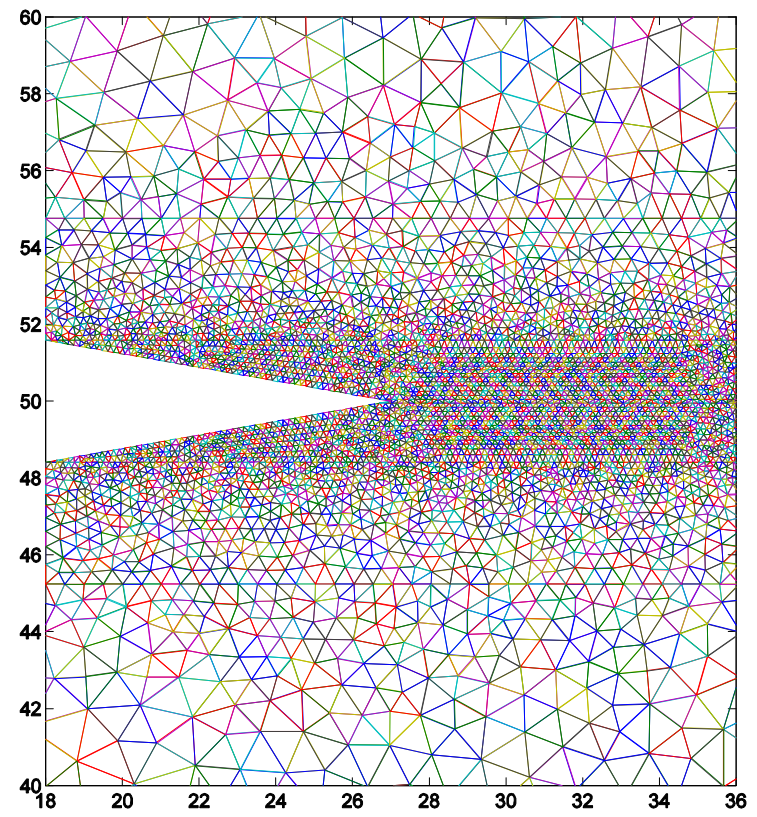

Figure 8: Detail of the mesh refinement for $2 \alpha=20 \mathrm{deg}$.

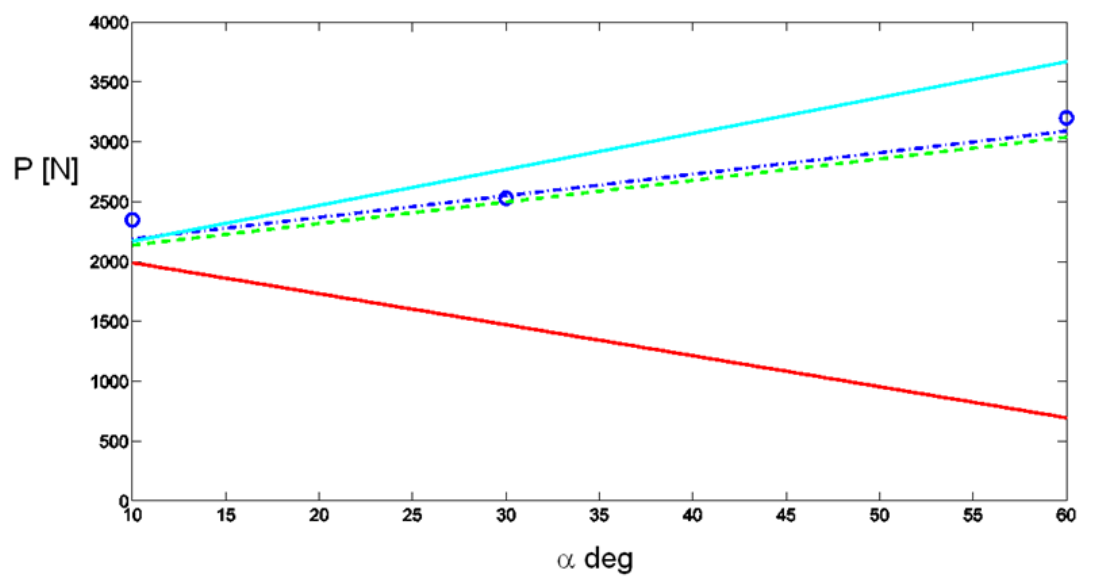

Figure 9: Profiles of the predicted failure loads for variable $\rho$ : divergence for $\rho=0.0001 \mathrm{~mm}$ (red dashed line), green dashed line: $\rho=0.01 \mathrm{~mm}$, blue dashed dotted $\rho=0.0075 \mathrm{~mm}$, ciano continuous line: $\rho=0.002 \mathrm{~mm}$; circles indicate experimental results.

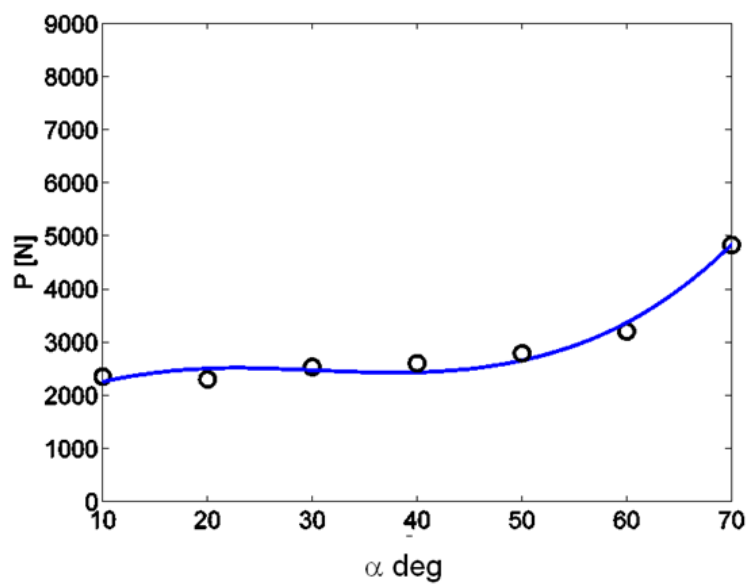

Figure 10: Comparison of the static failure loads obtained with the present approach (blue continuous line) with the experimental results presented in [2]. 
For an opening angle $2 \alpha=20 \mathrm{deg}$, Fig. 11 displays the map of the total stress component $\sigma_{y y}$ in a deformed configuration, which was obtained by using a magnification factor equal to 100. In Figs. 12 and 13, respectively, the total stress map and the profiles of the relevant stress components are reported. In particular, the black cross markers indicate the values of the total stress component at the Gauss points placed along the crack line, while the regularized stress $\sigma_{\rho}^{c}$ are marked with red dots. It can be noted that the total strain attains its maximum value $105 \mathrm{MPa}$ at the notch tip. Because, the enrichment extends over a length of $3 \mathrm{~mm}$ beyond the notch tip, a new stress singularity is there detected. An extension of the present approach to other non-linear constitutive laws is possible. However, in XFEM based approaches, in general, a crack tracking criterion has to be adopted in order to avoid that secondary multiple cracks open. This is a requirement common to embedded discrete-crack approaches. In the implicit gradient approach, instead, no hypotheses on the position of the critical points are necessary, because the effective stress is computed by post-processing the results, which are obtained by means of any standard finite element formulation of a continuum model.

An advantage of the present approach over existing cohesive models is that, here, the bulk constitutive law of the material can be directly assumed, because the process zone behavior is not modeled by means of a traction-separation law. Instead, the stress and strain fields $\boldsymbol{\sigma}_{\rho}^{c}$ and $\boldsymbol{\varepsilon}_{\rho}^{c}$ are introduced, which can be modeled with same constitutive law of the bulk.

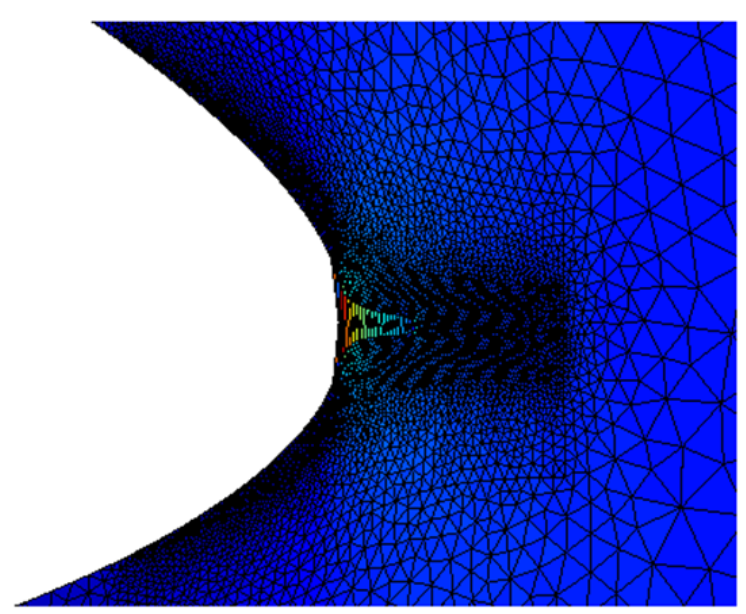

Figure 11: Stress map in the deformed mesh for $2 \alpha=20$ obtained with a magnification factor 100.

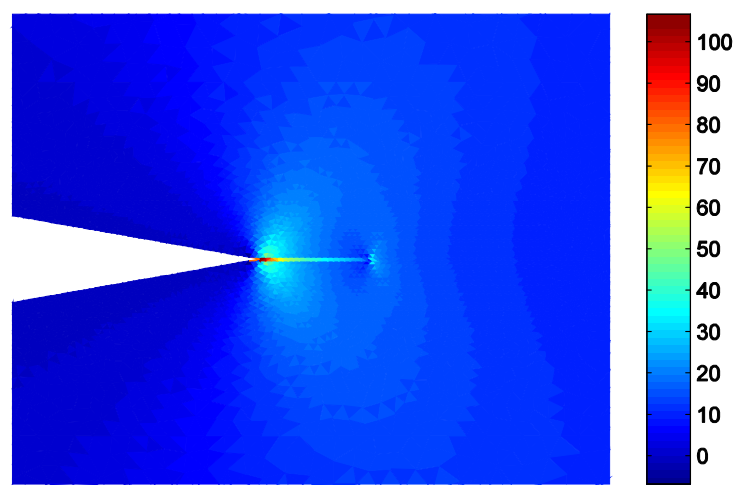

Figure12: Total stress map for $2 \alpha=20$.

\section{CONCLUSIONS}

$\mathrm{I}$ $\mathrm{n}$ the present note, a new approach based on a length-enriched eXtended Finite Element Method (XFEM) has been applied to the evaluation of the failure loads of tensile PMMA specimens. In particular, a comparison with the experimental results presented by Seweryn [2] has been performed. The present analysis shows that the proposed 
method makes it possible not only to avoid stress singularities but also to lead to a satisfying approximation of the experimental failure loads. In order to fully assess the effectiveness of the proposed method, further investigations are required, such as the study of mixed loading modes. The present approach has the same potential of cohesive models without the need of inserting the crack line along the element boundaries. Moreover, it can be easily used to deal with general non-linear constitutive laws.

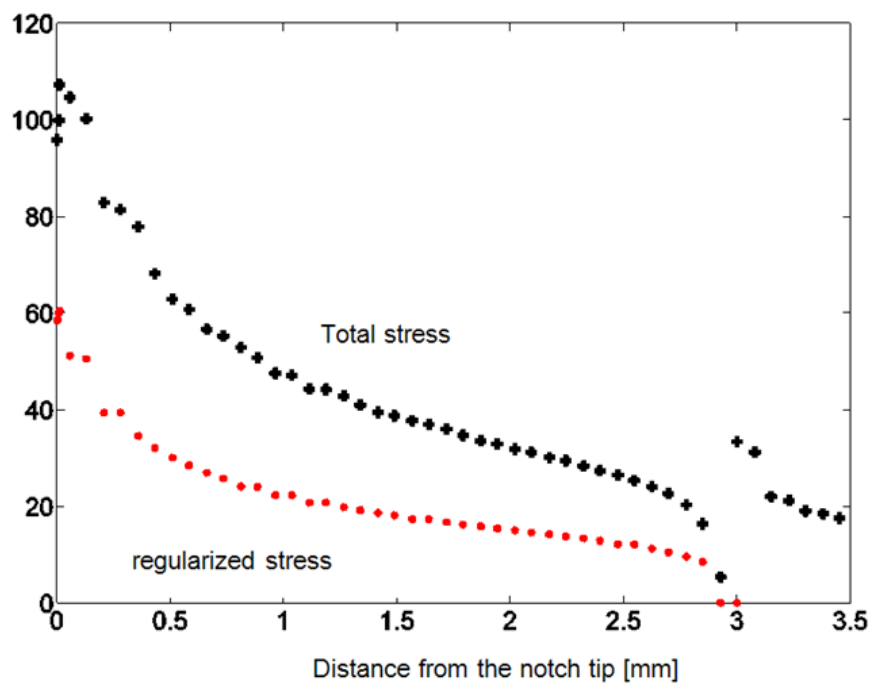

Figure 13: Profiles of the total stress field and the regularized stress $\sigma_{\rho}^{c}$ along the crack line for $2 \alpha=20 \mathrm{deg}$.

\section{REFERENCES}

[1] H. Neuber, Konstruktion, 20 (1968) 245.

[2] A. Seweryn, Engineering Fracture Mechanics, 47 (1994) 673.

[3] L. Susmel, D. Taylor, International Journal of Fatigue, 28 (2006) 417.

[4] D.G.B. Edelen, Continuum Physics, Vol. IV Academic Press, New York, (1976) 75.

[5] C.A. Eringen, D.G.B. Edelen, International journal of Engineering Science, 10 (1972) 233.

[6] R. H. J. Peerlings, M.G.D. Geers, R. de Borst, W.A.M. Brekelmans, Int. J. Solids Structures, 38 (2001) 7723.

[7] R. Tovo, P. Livieri, E. Benvenuti, International Journal of fracture, 141 (2006) 497.

[8] R. Tovo, P. Livieri, Engineering Fracture Mechanics, 74 (2007) 515.

[9] F.J. Gomez, M. Elices, Engineering Fracture Mechanics, 70 (2003) 1913.

[10] J. Planas, M. Elices, G. V. Guinea,F. J.Gómez, D. A. Cendón, I. Arbilla, Engineering Fracture Mechanics, 70 (2003) 1759.

[11] A. Cornec, I. Scheider, K. H. Schwalbe, Engineering Fracture Mechanics, 70 (2003) 1963.

[12] N. Moes, T. Belytschko, Engineering Fracture Mechanics, 69 (2002) 813.

[13] B. L. Karihaloo, Q. Z. Xiao, International Journal of Fracture, 150 (2008) 55.

[14] X. Y. Liu, Q. Z. Xiao, B. L. Karihaloo, International Journal for Numerical Methods in Engineering, 59 (2004) 1103.

[15] J. Réthoré, S. Roux, F. Hild, International Journal for Numerical Methods in Engineering, 81 (2010) 269.

[16] H. Mihashi, N. Nomura, Nuclear Engineering and Design, 165 (1996) 359.

[17] E. Benvenuti, Computer Methods in Applied Mechanics and Engineering, 197 (2008) 4367.

[18] E. Benvenuti, A. Tralli, G. Ventura, International Journal for Numerical Methods in Engineering, 74 (2008) 1097.

[19] E. Benvenuti, Mesh-size-objective XFEM for regularized continuous/discontinuous transition, accepted for publication on Finite Element Analysis and design (2011). 